\title{
Automated identification of insect vectors of Chagas disease in Brazil and Mexico: The Virtual Vector Lab
}

\author{
Rodrigo Gurgel-Gonçalves ${ }^{1}$, Ed Komp ${ }^{2}$, Lindsay P Campbell ${ }^{3}$, Ali Khalighifar ${ }^{3}$, Jarrett Mellenbruch ${ }^{4}$, \\ Vagner José Mendonça ${ }^{1}$, Hannah L Owens ${ }^{3,5}$, Keynes de la Cruz Felix ${ }^{6}$ ，A. Townsend Peterson ${ }^{\text {Corresp., }}{ }^{3}$, Janine \\ M Ramsey ${ }^{6}$ \\ 1 Faculty of Medicine, Universidade de Brasília, Brasilia, DF, Brazil \\ 2 Information and Telecommunication Technology Center, University of Kansas, Lawrence, Kansas, United States \\ 3 Biodiversity Institute, University of Kansas, Lawrence, Kansas, United States \\ 4 Spencer Art Museum, University of Kansas, Lawrence, Kansas, United States \\ 5 Florida Museum of Natural History, University of Florida, Gainesville, Florida, United States \\ ${ }^{6}$ Centro Regional de Investigación en Salud Pública, Instituto Nacional de Salud Publica, Tapachula, Chiapas, Mexico
}

Corresponding Author: A. Townsend Peterson

Email address: town@ku.edu

Identification of arthropods important in disease transmission is a crucial, yet difficult, task that can demand considerable training and experience. An important case in point is that of the 150+ species of Triatominae, vectors of Trypanosoma cruzi, causative agent of Chagas disease across the Americas. We present a fully automated system that is able to identify triatomine bugs from Mexico and Brazil with accuracy consistently above $80 \%$, and with considerable potential for further improvement. The system processes digital photographs from a photo apparatus into landmarks, and uses ratios of measurements among those landmarks, as well as (in a preliminary exploration) two measurements that approximate aspects of coloration, as the basis for classification. This project has thus produced a working prototype that achieves reasonably robust correct identification rates, although many more developments can and will be added, and-more broadly-the project illustrates the value of multidisciplinary collaborations in resolving difficult and complex challenges. 


\section{Automated identification of insect vectors of Chagas disease \\ 2 in Brazil and Mexico: The Virtual Vector Lab}

3 Rodrigo Gurgel-Gonçalves ${ }^{1}$, Ed Komp ${ }^{2}$, Lindsay P. Campbell ${ }^{3}$, Ali Khalighifar ${ }^{3}$, Jarrett

4 Mellenbruch $^{4}$, Vagner José Mendonça ${ }^{1}$, Hannah L. Owens ${ }^{3,5}$, Keynes de la Cruz Felix ${ }^{6}$,

5 A. Townsend Peterson ${ }^{3}$, Janine M. Ramsey ${ }^{6}$

$6 \quad{ }^{1}$ Faculty of Medicine, Universidade de Brasilia, Brasilia, DF, Brazil

7 2Information and Telecommunication Technology Center, University of Kansas, Lawrence,

8 Kansas, United States

$9 \quad{ }^{3}$ Biodiversity Institute, University of Kansas, Lawrence, Kansas, United States

$10 \quad{ }^{4}$ Spencer Art Museum, University of Kansas, Lawrence, Kansas, United States

$11{ }^{5}$ Florida Museum of Natural History, University of Florida, Gainesville, Florida, United States

$12{ }^{6}$ Centro Regional de Investigación en Salud Pública, Instituto Nacional de Salud Publica,

13 Tapachula, Chiapas, Mexico

\section{Abstract}

15 Identification of arthropods important in disease transmission is a crucial, yet difficult,

16 task that can demand considerable training and experience. An important case in point

17 is that of the 150+ species of Triatominae, vectors of Trypanosoma cruzi, causative 
18 agent of Chagas disease across the Americas. We present a fully automated system

19 that is able to identify triatomine bugs from Mexico and Brazil with accuracy consistently

20 above $80 \%$, and with considerable potential for further improvement. The system

21 processes digital photographs from a photo apparatus into landmarks, and uses ratios of

22 measurements among those landmarks, as well as (in a preliminary exploration) two

23 measurements that approximate aspects of coloration, as the basis for classification.

24 This project has thus produced a working prototype that achieves reasonably robust

25 correct identification rates, although many more developments can and will be added,

26 and-more broadly - the project illustrates the value of multidisciplinary collaborations in

27 resolving difficult and complex challenges.

\section{Introduction}

29 The challenge of identifying insects important in public health or agriculture is significant,

30 since efficient surveillance and mitigation often depend on non-expert or community

31 participation, and yet often must focus on particular species (e.g., Dias et al. 2002).

32 Dichotomous keys have long been the primary tool for most taxonomic identifications,

33 although their use is limited by the expertise required (Gaston 1992, Drew 2011).

34 Alternatives, such as creation of cooperative extension programs, as at state and federal

35 land-grant institutions across the United States (Allen and Rajotte 1990), are expensive

36 in terms of resources and personnel; recent approaches creating online field guides

37 have made identification more efficient, but again are labor-intensive (Stevenson et al.

38 2003). DNA barcoding has also been explored as a substitute for morphology-based 
39 identification, but its cost and lack of efficacy preclude its use outside research or

40 emergency efforts (Hebert and Gregory 2005, Meier et al. 2006).

41 The possibility of automating the identification process has long been discussed and 42 explored (Weeks et al. 1997, Gaston and O'Neill 2004, MacLeod 2007, MacLeod et al. 43 2010). Progress has been made in using acoustic or other waveforms to identify some 44 taxa (e.g., birds, Orthoptera; Moore and Miller 2002, Chesmore 2004, Acevedo et al. 45 2009), and the Digital Automated Identification SYstem (DAISY) has been used with 46 some success based on optical imagery in several insect groups (Weeks et al. 1997, 47 Weeks et al. 1999, Watson et al. 2003). Certain contributions have focused on improving 48 the algorithms and approaches to identification (Schroder et al. 1995, Weeks et al. 1997, 49 Kang et al. 2012a, Kang et al. 2012b, Wang et al. 2012a, Wang et al. 2012b), whereas 50 others have made steps toward more full automation of the process (Arbuckle et al. 51 2001, Mayo and Watson 2007, Yang et al. 2015).

52 Progress in automating identification of medically important vectors, however, has been 53 slower. Although some advances have been made toward automated identification of 54 mosquitoes (Culicidae) using wing venation (Zhou et al. 1985, Lorenz et al. 2015), more 55 effort has centered on DNA barcoding as a solution (Cywinska et al. 2006, MBI 2009). 56 For sand flies (Psychodidae), the only steps towards automating identification have

57 been via mass-spectrophotometry of proteins (Mathis et al. 2015) and DNA barcoding 58 (Pinto et al. 2015). Automated identification of ticks (Ixodidae and Argasidae) and kissing 59 bugs (Triatominae) has not been attempted, although many morphometric studies have 60 been developed (e.g., Voltsit and Pavlinov 1995, Dujardin and Slice 2007), and online 
61 dichotomous keys have now been implemented to provide some level of identification

62 support at least in Brazil (http://triatokey.cpqrr.fiocruz.br/).

63 Here, we present a fully-automated visual identification system for kissing bugs

64 (Triatominae: Hemiptera: Reduviidae; full nomenclatural details for each species are

65 provided in tables 1 and 2), vectors of Trypanosoma cruzi, the etiologic agent of Chagas

66 disease. Beginning with a high-quality photographic image, we have automated all steps

67 from preparing and processing the image to identifying landmarks and running and

68 interpreting identification routines. We implement this system for the triatomines of Brazil

69 (39 species) and Mexico (12 species, including the 3 haplogroups of the Triatoma

70 dimidiata complex), but are already broadening the scope of the project to all

71 triatomines, and are exploring the transfer of the techniques employed to other medically

72 important arthropod groups.

\section{The Automatization Workflow}

74 Photographs for input

75 We observed early in the course of this project that the triatomine photographs available

76 to us were of variable, and generally low, quality. As a consequence, we set about

77 designing an apparatus that would permit taking consistent, high-quality, repeatable

78 photographs of triatomines at low cost (Figure 1). It is constructed of acrylic polymer and

79 alumina trihydrate (commercially available as Corian®), with metal fittings and a metal

80 support rod. The upper platform of the apparatus has a cradle in which an iPod touch $\circledast$

81 (or cell phone) is nestled, as well as a ring of LED lights to illuminate the bug for

82 photography. The lower platform includes a size standard, as well as a pin point on 
83 which the specimen can be affixed by impaling through the abdomen, such that the pin

84 is not visible in the resulting dorsal-view image. The lower platform is an even blue color,

85 which is an important feature for image processing (see below).

86 The iPod used for photography is equipped with an Olloclip 7x macro lens to optimize

87 close-range photography. The $5^{\text {th }}$-generation iPod generates $5 \mathrm{MP}$ images, whereas the

$886^{\text {th }}$-generation iPod delivers 8MP images. Photos are then uploaded to a DropBox folder

89 for communication to the processing and identification modules, a step that is soon to be

90 automated so that contribution of photos to the project is automatic. The iPod includes

91 videos in Portuguese (https://vimeo.com/187498921/e4ef52cd46), Spanish, and English,

92 showing how to use the apparatus. In future iterations of development of these

93 functionalities, we will take advantage of the GPS geotagging functionality of the iPod to

94 add geographic information automatically to photos and data records, although care

95 must be taken either to match the geographic coordinates of the photo with the locality

96 where the specimen was collected or to provide an independent georeference.

97 The apparatus was used to assemble a large reference collection of photos for as many

98 triatomine species that occur in Brazil and Mexico as was available for testing these

99 approaches. Triatomines were photographed from collections from across Brazil

100 (Universidade de Brasília, FIOCRUZ Bahia, FIOCRUZ Rio de Janeiro, FIOCRUZ Minas

101 Gerais, Universidade Estadual Paulista) and Mexico (Centro Regional de Investigación

102 en Salud, Instituto Nacional de Salud Pública México; Laboratorio Estatal de Salud

103 Pública de Guanajuato; Universidad Autónoma Benito Juárez, Oaxaca; Universidad

104 Autónoma de Nuevo León, Monterrey). Only via the kind collaboration of colleagues at 
105 these institutions was it feasible to assemble the set of photographs necessary for this 106 work.

107 Our protocol for capturing reference images, which also represents best practices for

108 unknown bugs to be identified, is as follows. If bugs are preserved in ethanol, they are

109 allowed to dry for $10 \mathrm{~min}$; if dried, specimen pins and labels were removed whenever

110 possible to reduce bias in color measures of the dorsum. If specimens are from

111 insectaries (generally no more than two generations of captive propagation) or recent

112 field collections, they are killed by freezing for 15 minutes, cleaned with a damp brush,

113 allowed to dry for a few minutes, and placed on the pin point in the photo apparatus.

114 When possible (i.e., the specimen is not needed as a permanent specimen voucher),

115 the first pair of legs is removed to simplify image processing. The specimen is

116 photographed with the following steps: (1) size standard magnet positioned next to the

117 specimen with its upper surface flush with the specimen's dorsum (back); (2) LED light

118 ring turned on, along with the camera application on the iPod, 7X magnification lens

119 attached; (3) specimen centered in the picture frame, attempting to fill $>90 \%$ of the field

120 with the specimen's length; (4) lens focused and light meter reading checked; (5) photo

121 taken and quality checked on the iPod; (6) images uploaded to DropBox and quality

122 checked again. Photography takes $\sim 1$ minute per specimen.

\section{Image preparation and processing}

124 The photographs produced using the apparatus described above are quite consistent in

125 orientation, resolution, and quality, which enables full automation of their processing.

126 The sequence is illustrated in Figure 2: major steps include removing (digitally)

127 background and extraneous body parts (legs, antennae), orientation and identification of 
128 landmarks, measurements and calculation of ratios, and submission to identification

129 routines. Descriptions of our processing steps follow.

130 Correct image and extract the core body from the raw image._A first step in processing

131 images is to correct the image for lens distortion. The macro lens attachment to the iPod

132 introduces significant "pincushion" distortion to images. Since all photos are taken with

133 the same lens arrangement, and given only minor variation in the distance from lens to

134 target, a correction is applied using a fixed set of parameters in the plug-in lens-

135 distortion module of GIMP, an open-source Photoshop-like application. The two

136 parameters set are main $=12.0$, which specifies how much spherical correction to

137 introduce, and edge $=6.0$, which indicates the amount of additional spherical correction

138 at image edges.

139 A second step is to remove the background from the image. The photo apparatus

140 includes a baseplate with a solid blue color, which greatly facilitates isolating the sample

141 in the photograph. However, significant variation in the background color exists among

142 and even within images, owing to variation in ambient lighting, shading, etc. This effect

143 is particularly noticeable and critical near the edge of the bug's body. Hence,

144 background pixels are identified not as specific RGB colors, but by a relation among

145 RGB values, as follows: for each of the three color dimensions ( $R, G$, and $B)$, a pixel is

146 considered as background $B>0.75$ and $R<0.7 B$ and $G<0.9 B$, or if $B>0.65$ and $(R+$

$147 \mathrm{G}$ ) $<1.25 B$ and $1.5 \mathrm{R}<\mathrm{G}$. The first condition is that which most often is applicable (i.e.,

148 blue is relatively large and dominates red and green, to avoid including other bright

149 colors or white; particular values were chosen empirically, based on numerous trials). 
150 A third step identifies the specimen's body edge. An edge trace is extracted that satisfies

151 several properties: (1) the edge is divided into left and right portions; (2) the edge is

152 continuous such that each edge pixel is adjacent to two other edge pixels; (3) the edge

153 is exactly one pixel wide such that for any edge pixel, exactly 2 of its 8 neighbors are

154 also part of the edge; (4) the edge progresses monotonically in the y-direction (i.e.,

155 sagittally). These restrictions make for efficient operations, such as following edges,

156 detecting changes in direction, or identifying notable features. If one draws a horizontal

157 line across the specimen image, it intersects the edge at exactly two points: one on the

158 left side and one on the right side of the body.

159 The fourth step is to clip the legs and antennae from the image. Appendages appear as

160 relatively narrow, straight segments. Detecting such segments is a two-step process.

161 First, the binary image is traversed row-by-row and then column-by-column, recording

162 narrow bands of bug as sequences of zeroes (0) of length less than the maximum

163 segment width. Adjacent bands that form segments are collected for removal. Because

164 this procedure is applied first in the horizontal direction, and then in the vertical direction,

165 removal is best for appendages aligned horizontally or vertically; it can handle

166 appendages oriented at intermediate angles, so long as the width of the horizontal or

167 vertical slice is less than the maximum width parameter.

168 Finally, triangles left by previous clipping operations are smoothed. The appendages

169 removed in the previous step are sliced off along a vertical or horizontal line where the

170 appendage attaches to the body. If the edge at the attachment point is not exactly

171 horizontal or vertical, an artificial triangular shape is generated along the edge. A pass

172 along the edge identifies these triangular shapes as $90^{\circ}$ changes in direction; when such 
173 changes are detected, the two adjoining horizontal and vertical segments are replaced

174 by the hypotenuse connecting their endpoints.

175 Detect landmarks on body edge

176 A specialized detection function was developed for each landmark, as is described in

177 detail below, in general not depending on detection of other landmarks; left/right

178 landmarks (e.g., left eye center and right eye center) are detected as pairs. These

179 algorithms assume an approximate vertical line of symmetry, and use this assumption to

180 improve detection. Comparisons with other landmark positions are used in validation

181 steps, which may cause iteration of certain functions using additional constraining 182 arguments.

183 To locate the anterior and posterior extrema (clypeus and final tergits or wing tips,

184 respectively) of the dorsal view of each specimen, the points along the edge with 185 maximum and minimum $y$-values are identified. The point at the anterior extreme of the

186 head (clypeus) relies on correct edge extraction at the head and neck juncture, and

187 correct antenna exclusion. If the wings extend beyond the final tergits, then the point at

188 the posterior extreme of the specimen will be beyond the end of the abdomen. We note

189 that distinguishing between wing tips and final tergits as comprising the posterior

190 extreme of the specimen is a challenge for future development of this tool, and may

191 reduce measurement variation and improve identification in very useful ways.

192 The eye center and radius are identified next. The eyes are the most distinctive features

193 of the bug, appearing as hemispheres; no other portion of the edge has this shape. The

194 Circle Hough Transform is a well-known technique in digital image processing for 
195 detecting circles of arbitrary radius (Ballard 1981). However, for our purposes, to reduce

196 processing time and avoid false positives, a valid range of eye radius values for each

197 image is identified. First, the expected eye radius is estimated, and the search is

198 restricted to hemispheres with radii close to this estimate. An estimate of the eye radius

199 is core body length / 48 (estimated empirically); hence circles with radius in the range of

200 the estimate \pm 10 pixels are sought. Providing a radius range parameter to the transform

201 significantly reduces execution time, and avoids many false positives.

202 The Hough Transform returns many possible matches, but left and right hemispheres 203 that constitute a reasonable hypothesis of the pair of eyes for the bug are the target. As

204 such, in a series of conditionals and empirically-tuned exploratory filters, the following

205 conditions are imposed: (1) left and right eyes should be in approximately the same

206 position vertically; (2) the two eyes should have nearly the same radius; and (3) the eye

207 centers should be close to one another. An internal scoring scheme identifies the pair of

208 putative eyes with the highest score, but also requires a minimum match score. If no pair

209 scores above this minimum, the detector returns "NotFound," rather than values that are

210 likely incorrect. This approach detects left and right eye pairs, but can be confused if one

211 eye is obscured in an image, in which case the routine fails to detect either eye. In future

212 versions, we hope to develop single-eye detectors to provide at least some useful

213 information from lower-quality images or images of damaged individual specimens.

214 The next landmark to be located is the antenna junction. To this end, the routine

215 searches below the anterior extremus of the specimen, which typically is a relatively

216 straight vertical line segment, seeking the first significant horizontal deviation in

217 direction. This position is compared to the eye position on the body side: if it appears at 
218 or below the eye, the location is rejected. A secondary search routine uses the position

219 of the antenna junction on the opposite body side for guidance.

220 A major challenge in locating landmarks focuses on locating the pronotum fore lobe,

221 humerus, and midpoints. Because of the restriction that the extracted edge increases

222 monotonically in the $y$ dimension, the anterior-most tips of the pronotum fore lobe may

223 be clipped. Given the extracted edge, the anterior lateral margin of the pronotum fore

224 lobe appears as a dramatic directional change along the edge. Along the collar, the edge

225 is quite horizontal, but it turns downward at a relatively steep and constant angle at the

226 margin, which is the focus of our detection efforts. The posterior lateral margin

227 (humerus) of the pronotum can be identified similarly: the humerus widens at a relatively

228 constant angle until it attains its maximum width, at which point the edge angle changes,

229 again allowing detection. These paired edge points are used to detect midpoints

230 between left and right sides. Finally, maximum body width is extracted by simply

231 scanning the core body image row by row to find the maximum difference between the

232 right and left edges; the accuracy of this measure depends on the accuracy with which

233 the legs have been clipped off in earlier steps.

234 Other features that used in our analyses are extracted and characterized as follows.

235 Body area is summarized as the area of the head, pronotum, and abdomen, comprising

236 the entire white area in the fourth panel of Figure 2. The head area is the portion of the

237 body core anterior to the pronotum fore lobe. The pronotum area (approximate) is the

238 portion between the fore lobe and humerus angle landmark pairs. Abdomen area is 239 derived by subtraction. 
Classify sample

241 The landmarks described above are then turned into a set of descriptors to be used in

242 the identification exercises. Of course, it is possible to compute large numbers of

243 measurements for these uses: distances among landmarks, angles between different

244 line segments connecting landmarks, ratios of pairs of measurements, etc. However,

245 because more is not always better, a first step was to select a small, but maximally

246 effective, set of features with which to distinguish among the triatomine species.

247 Three criteria are employed in the process of choosing the set of descriptors to a core

248 suite for identification: (1) The average of each pair of symmetric measurements is used

249 as a single feature, which reduces minor, random error in automatic detection of

250 landmark locations. (2) Taxonomic research on triatomines was reviewed to detect and

251 quantify salient and particularly relevant features. (3) Extensive exploratory testing was

252 done with different combinations of the candidate features.

253 All measurements are made in terms of pixel units on the digital image; for size-related

254 measurements explored later in this study, we use size-standard information to convert

255 to absolute measurements $(\mathrm{mm})$. To avoid problems of scaling, and of size variation

256 related to individual age, a series of ratios of pairs of measurements is used, such that

257 classification features are unitless. The features chosen were thus as follows: total

258 length / clypeus - pronotum forelobe midpoint, total length / mean lateral eye margin,

259 total length / mean eye center, total length / mean lateral pronotum forelobe, total

260 length / pronotum forelobe midpoint to pronotum-humeral angle midpoint, total length /

261 mean lateral pronotum humeral angle, total length / total area, total length / maximum

262 body width, and total length / mean eye center - mean lateral pronotum fore lobe. 
263 To explore the utility of interior features (i.e., within the outline of the body), and more in 264 particular the possible utility of characteristics of coloration, we examined one species

265 pair that proved problematic to identify in initial analyses: T. pallidipennis and T.

266 phyllosoma. The triatomine experts on the project team indicated that these two species

267 are easily differentiated by the color of the corium: in T. pallidipennis, it is mostly white,

268 whereas in T. phyllosoma, it is black with two light spots. Hence, a rectangle bounded by

269 the pronotum humerus edges (which are already landmarked), extending posteriorly

270 twice the length of the pronotum is extracted. Within the rectangle, a triangular area

271 roughly corresponding to the scutellum is identified. Finally, the ratio of the average

272 gray-scale value within the rectangle and outside the triangle to the average grayscale

273 value inside the triangle is calculated; this ratio is used as a further characteristic of the

274 bugs that could be included in identification exercises. This ratio is quite high in $T$.

275 pallidipennis, and low in T. phyllosoma.

276 While working on color variation around the scutellum, we noticed pattern variation on

277 the pronotum among various species. For many species, the pronotum has a relatively

278 constant color, but $T$. brasiliensis shows a series of stripes. For T. pintodiasi and $T$.

279 carcavalloi, the upper portion of the pronotum is relatively dark, and the lower portion is

280 much brighter. Instead of attempting to describe the actual patterns, and developing

281 detection algorithms for each, we explored a very simple summary measurement. We

282 record the color variation among the pixels of the pronotum.

283 Finally, based on knowledge on the part of triatomine specialists on the team, we 284 perceived the utility of exploring measures of absolute size. As a step to this end, we 
285 used total length, but translated into absolute measurements $(\mathrm{mm})$ via reference to the

286 size of the size standard (which was detected and measured using routines for detecting

287 circular structures described above). This single absolute-size measure proved useful in

288 improving classification of some difficult species pairs.

289 A key observation from initial data analyses was the improvement of identifier

290 performance using smaller pools of species from among which the classifier chooses.

291 The use of pairwise combinations of binary classifiers for multi-class problems is well-

292 described in the literature (Wu et al. 2004). For classification, our routines use the nnet

293 package in the language R (https://cran.r-project.org/web/packages/nnet/) which

294 implements a feed-forward neural network model (Ripley 1996) These routines require

295 few parameters, relying instead almost exclusively on the data provided for model

296 calibration. To construct binary classifiers for every pair of species, among 39 species

297 from Brazil $(39 \times 38) / 2=741$ and 12 species from Mexico $(12 \times 11) / 2=66$ such

298 classifiers exist, and a simple voting scheme is used to combine results, such that the

299 species name chosen by the most pairwise classifiers is selected. This pairwise

300 combination of binary classifiers is more effective than a single multi-class classifier,

301 particularly when the target pool is restricted to those species represented in the fauna

302 predicted by ecological niche models to be present at that site (see next section).

\section{Identify faunal subsets based on modeled distributions}

304 To reduce suites of species under comparison (see below), it was necessary to

305 summarize the geographic distributions of the 39 Brazilian and separately the 12

306 Mexican triatomine species of interest in our identification efforts. Using ecological niche

307 modeling techniques and data sources described in detail elsewhere (Gurgel-Gonçalves 
308 et al. 2012, Ramsey et al. 2015), we created binary maps summarizing potential

309 geographic distributions for each of the 39 Brazilian and 12 Mexican species. Ecological

310 niche models were drawn from our previous publications (Gurgel-Gonçalves et al. 2012,

311 Ramsey et al. 2015) for 14 species. For 17 more species not included in the earlier

312 publications, we developed new ecological niche models following the same protocols

313 as in those publications. We converted them to binary maps using an adjusted least

314 training presence threshold approach, in which we sought the highest cut-off that

315 included $(100-E) \%$ of the calibration data, where $E$ is the percentage of occurrence

316 data likely to include meaningful errors (Peterson et al. 2008) and was set at $5 \%$. For the

317 remaining 20 species, for which $<10$ occurrence points were available, and robust model

318 creation using the methods of the previous publication were not possible; we instead

319 used a simple known-extent-of-occurrence approach to delineate distributional areas of

320 potential distributions. A $20 \mathrm{~km}$ buffer was created around the known occurrence points

321 of each species, and considered all sites within that buffer as within the distributional

322 area for the species.

323 Resulting binary distribution maps of each species were stacked using the raster

324 package in $\mathrm{R}$ (Hijmans 2015). Values within each grid cell were extracted to create a

325 presence/absence matrix (PAM) with columns representing individual species and rows

326 representing all $5 \times 5 \mathrm{~km}$ grid cell locations across Brazil and Mexico separately (Arita et

327 al. 2008, Soberón and Ceballos 2011). We identified a random sample of 5000 rows

328 from the PAM to choose random species combinations across Brazil and Mexico

329 (separately) among which to test our classifiers based on real-world combinations of

330 triatomine species (Figure 3). 
331 Identifier testing

332 Given the limited number of samples (photos) for several of the species, we used a

333 leave-one-out cross-validation approach for identifier testing, which maximizes the

334 number of species that can be included. We use this testing approach as we are still in

335 the process of building and testing the technology that comprises this tool. Eventually,

336 once we create a complete reference set of images, all of the reference images can be

337 used to create identifiers that can be used for any new test images.

338 To test identification abilities among species in local fauna sets, the input data are 339 restricted to those species appearing in the local fauna, and training and testing of

340 classifiers proceed as for the full set of species. To assess the related question of

341 random species combinations (i.e., sets of species smaller than the whole 39- or 12-

342 species set, but without the local-fauna constraint), we used the function "sample" in R

343 to select random collections of specified numbers of species from the 39 Brazilian and

34412 Mexican species to generate virtual species lists. For each number of species, we

345 generated 200 such random faunas. For visualization of the spread of the different

346 species in morphological spaces, we conducted a principal components analysis of the 347 morphometric measurement matrix.

348 All of the program code and data on which this report is based is freely and openly

349 available, in the hope that our work so far can provide a foundation of high-quality data

350 inputs and progress-to-date in programming. Program code and the raw photographs of

351 triatomines are available from the Dryad Digital Repository:

352 http://dx.doi.org/10.5061/dryad.br14k; the data on which the final figure is based are 353 available in the Supplementary Materials. 


\section{Results}

355 We had available to us a total of 1903 images of 67 Brazilian species and 428 images of

35619 Mexican triatomine species (Table 1; see Supplementary Materials). Since few

357 images were available for 28 of the Brazilian species and 7 of the Mexican species,

358 efforts were concentrated on 39 Brazilian and 12 Mexican species for which sufficient

359 images were available (12 in all cases, except Panstrongylus rufoturberculatus in

360 Mexico, for which only 7 were available). Automated morphological measurements from

361 these images $(N=1502$ for Brazil and $N=396$ for Mexico) and the landmarks extracted

362 therefrom showed considerable spread in each country, suggesting that different species

363 present measurably different shapes that can serve as an effective base for identification

364 exercises (Figure 4).

365 In general, our classification efforts resulted in successful identifications of triatomine

366 specimens. Even in the extreme challenge of identifying an image of a bug from among

367 all species for which we had sufficient numbers of images, our average identification

368 success rate (i.e., average of species averages) was $87.8 \%$ for Brazilian and $80.3 \%$ for

369 Mexican species. We note that inclusion of two color characteristics improved the

370 correct identification rate for T. pallidipennis from $69.8 \%$ to $90.7 \%$. Indeed, this color

371 characteristic alone improved overall correct identification rates even among the 39

372 Brazilian triatomines from $78.4 \%$ to $84.0 \%$. Addition of the absolute-size characteristic

373 (total length) provided further improvement of overall correct identification rates, reading

374 the final rates of $80.3 \%$ (Mexico) and $87.8 \%$ (Brazil). 
375 The basic result is encouraging, as it indicates that sufficient information exists in the 376 variation among species in shape to support automated identification, and that color377 and size-related features may allow significant improvements. Identification ability rose 378 notably when we moved to our reduced-fauna identification process (described below). 379 Confusion matrices for each country are presented in Supplementary Materials, which 380 shows how each image was classified correctly or incorrectly, and, in the latter case, for 381 which species was the image mistaken; overall success rates are presented in Tables 1 382 and 2.

383 Looking across the 39 Brazilian species, identification success rates ranged from $384100.0 \%$ (Eratyrus mucronatus, Panstrongylus diasi, Psammolestes tertius) to as low as $38558.8 \%$ (T. williami). For 7 species, identification success was $95 \%$ or above; for 18 386 species, success was $90 \%$ or above; and for 31 species, identification success was $80 \%$ 387 or above. The worst-classified species ( $T$. williami) was confused in 3 cases with $T$. 388 matogrossensis, in 2 cases with $T$. guazu, (and others with which single images are 389 confused); the second-worst-classified species ( $T$. guazu) was mistaken for 5 different 390 species. For Mexican species, identification success rates were lower, ranging from 391 100.0\% (Panstrongylus rufotuberculatus) to as low as $60.0 \%$ (T. nitida); for 3 species, 392 identification success was $90 \%$ or above; for 7 species, identification success was $80 \%$ 393 or above.

394 Exploring the effect of number of reference species on the success of identification 395 exercises, we rarefied the species from each country at random to produce smaller 396 numbers of species among which to discriminate (Figure 5). For Brazilian species, the 397 correct identification rate rose from $88.0 \%$ for 37 -species subsets to $99.4 \%$ for 2 -species 
398 subsets, in an approximately linear manner. This relationship translated into an

399 improvement of $0.335 \%$ in classification rate for every reduction of one species. For

400 Mexican species, identification success was uniformly lower $(80.7 \%$ for 11 species,

401 rising to $97.6 \%$ for 2-species subsets); curiously, the rate of improvement of identification

402 success with smaller faunas was higher, at $2.11 \%$ per reduction of one species (Figure 403 5).

404 A further question centered on the relationship between real-world sets of co-occurring

405 species versus the random subsets analyzed. We used our library of maps of

406 distributional areas to identify combinations of species potentially present at localities,

407 which ranged as high as 12 species in Brazil and 11 species in Mexico (Figure 3).

408 Correct identification rates among the real-world sets of species did not differ

409 significantly from the random reductions (Figure 5): that is, the same relationship

410 characterized real-world and random combinations.

411 Given the clear advantage of classifying images among smaller pools of species, the

412 biggest challenge for our methodology is when the species image submitted is not

413 among the pool among which one is testing — that is, when the presence of the species

414 is a surprise (e.g., poorly known species' distributions, human-mediated dispersal

415 outside of the species' distribution). On this front, we have encountered challenges-that

416 is, as a backup, we can simply identify images against the broader suite of all 39

417 species in Brazil, for which we have a correct identification rate of $87.8 \%$, though

418 numbers for Mexico are a more disappointing $80.3 \%$ among 12 species. We conducted

419 large numbers of tests, seeking some relationship between classifier outputs and 
420 whether or not the 'true' species is included in the pool, but have not encountered any

421 clear signal.

\section{Discussion}

423 This study presents "full" automation of the identification process for the Triatominae, a

424 medically important insect group. That is, with our system described above, once an

425 image is captured, all processing is automated and no human intervention is needed.

426 This level of autonomy distinguishes the Virtual Vector Lab from other such efforts

427 (Mayo and Watson 2007), and exists thanks to the multidisciplinary team that worked on

428 this project from diverse perspectives: visual art, computer science, insect taxonomy,

429 biogeography, etc. Some limitations still exist, and are reviewed in the next section.

430 Caveats._-Although our overall, country-wide success rates were above $80 \%$, our key to

431 success in getting relatively high correct identification rates is the creation of small pools

432 of candidate species from which to choose the answer, which we achieve via reference

433 to distribution maps for each of the triatomine species. That is, we have dedicated

434 considerable time and effort to the challenge of understanding geographic distributions

435 of each triatomine species in Brazil (Costa et al. 2002, Almeida et al. 2009, Gurgel-

436 Gonçalves et al. 2011, Costa and Peterson 2012, Gurgel-Gonçalves et al. 2012, Costa

437 et al. 2014) and Mexico (Ramsey et al. 2000, Beard et al. 2002, Peterson et al. 2002,

438 López-Cárdenas et al. 2005, Ibarra-Cerdeña et al. 2009, Ibarra-Cerdeña et al. 2014,

439 Ramsey et al. 2015). These mapping efforts, of course, are based on what information is

440 available, and-as such-can improve through time, particularly if the Virtual Vector Lab

441 is eventually tied to databases that archive the results of large numbers of identification

442 exercises for triatomines from many sites. 
443 The dimension of the identification challenge in which further effort and innovation are

444 needed is when the specimen to be identified is collected outside its predicted

445 distribution and therefore is not represented in the local-fauna pool of species. We

446 experimented at great length to address this problem, but were not successful in arriving

447 at a concrete solution. Two interesting features that were noted were as follows. Results

448 from pairwise species identification exercises were remarkably stable with regard to

449 which species were included in the identification challenge. Our majority-vote approach

450 to reconciling many individual species-by-species comparisons was usually won by a 451 single vote-i.e., often, two species 'tied' as the most likely identification, so the winning

452 vote was the direct, head-to-head comparison of those two species. Hence, it appears

453 that little information is available to guide the identification process other than the head-

454 to-head comparisons. Certainly, a good indicator of probable problems is when the local-

455 fauna identification and the global-fauna identification do not agree; we have

456 experimented with what to do when this "red flag" is raised, and have a number of

457 possible solutions (e.g., add the global-fauna choice to the local fauna list, and re-run

458 the estimator), but the success of this approach depends on the success rate of the

459 global identifier. This challenge will be a focus of our continuing efforts.

460 Where our identification routines tend to fail more frequently is — not at all surprisingly -

461 in comparisons within the species complexes that are known within the Triatominae

462 (Lent and Wygodzinsky 1979, Schofield and Galvão 2009). For example, within the

463 protracta complex in Mexico, our routines had great difficulty in differentiating T. barberi

464 from T. nitida. Within the phyllosoma complex, 6 species are included in this analysis,

465 and T. phyllosoma was the most often confused with complex-specific sister species, 
466 The three dimidiata complex haplogroups were as differentiated among themselves, as

467 with all other species, the closest belng the species morphologically most similar outside 468 the complex (T. mexicana).

469 Future steps.-For the moment, a constraint on the Virtual Vector Lab system is the 470 requirement for high-quality images of the bugs. That is, much of our initial processing is 471 possible thanks to the uniform blue background, the even lighting provided by our photo 472 apparatus, and the use of a macro lens. We expect that combining our ongoing 473 algorithm advancements with the rapidly increasing capabilities of cell-phone cameras 474 should first allow discontinuation of need for a macro lens and dedicated iPod, and 475 eventually of need for the photo platform apparatus itself, in successful analysis of 476 triatomine images. Such developments would empower fully non-expert and community 477 participation in development and use of the Virtual Vector Laboratory via any common 478 cell phone and a dedicated application. Development and testing of those functionalities 479 is a priority in the next phase of the project.

480 Another constraint on our present implementation is that of the initial assumption that 481 the photograph is indeed a triatomine as a starting point to identification exercises. 482 Distinguishing a triatomine from, say, a mosquito or a tick should be simple, but 483 comparisons with other reduviids will be much more challenging. The reason that we 484 chose triatomines as a first focal group for the Virtual Vector Lab is their size and 485 relatively flat, two-dimensional morphology. To distinguish triatomines from other 486 reduviids may require other views of the insect (antennae, legs, proboscis), although we 487 have not as-yet conducted the necessary tests. Similar concerns apply to juvenile life 488 stages: our present implementation is restricted to adults, the most likely stage in 
489 contact with humans. We may best deal with these challenges via instructions to users 490 that will allow them to eliminate non-triatomines a priori, but that will also be explored in 491 future phases of the project (Valdez-Tah et al. 2015).

492 A major source of optimism is that we have achieved very satisfactory correct 493 identification rates working almost exclusively with information on shape of the bugs, 494 which compares well with results of previous such efforts: $84 \%$ among 5 species of 495 papilionid butterflies by Wang et al. (2012a), 93\% among few species at the ordinal level 496 by Wang et al. (2012b), 85.7-100\% among 17 species of mosquitoes based on wing497 shape characters (Lorenz et al. 2015), and 90-98\% among 7 species of Neuroptera 498 (Yang et al. 2015). From the two-dimensional images available to us, we can, in theory, 499 take advantage of information on shape, size, and coloration. Using one of these three 500 realms of information (shape), with some additional information from coloration and size, 501 we have managed quite-useful accuracy in identifications, even among the three cryptic 502 haplogroups traditionally considered under the name T. dimidiata (HG1 75.0\%, HG2 $50380.0 \%$, HG3 82.5\%).

504 As an indication of the promise of still-better identification success that can come from 505 adding information on color to our classifiers, we explored the utility of a classifier 506 focused on the white patch on the corium of T. pallidipennis. Not only did this classifier 507 resolve most of the confusion that our previous classifier had encountered with 508 distinguishing between T. pallidipennis and T. phyllosoma (sister species), but indeed 509 the addition of one color-related metric in our data matrix improved our classification 510 success even for Brazilian triatomines by $3.2 \%$. We obtained similar improvements in 511 identification success upon including an absolute size measure. As such, in future 
512 efforts, we will explore more complete variable sets characterizing shape, size, and 513 coloration.

514 Recently evolved species within complexes are clearly providing the greatest challenges

515 for the system, even though the system is also giving us insights into shape and sister

516 clades. The three T. dimidiata haplogroups in Mexico, clearly very closely related

517 species, had previously been recognized based on molecular evidence, yet our

518 morphometric analyses resulted in identifications with accuracy similar to that among

519 other North American complexes. As such, we see great promise in a next generation of

520 classifiers that will integrate morphometrics with characteristics corresponding to the

521 interior of the body of the triatomines (as with the scutellum), and particular to the

522 relative coloration of different portions of the body. We are also optimistic that specific

523 classifiers and metrics can be built for each species pair that presents challenges to the

524 more generic classifier that we have described in this paper.

525 Finally, extension of these general approaches to other groups is eminently feasible.

526 Certainly, two-dimensional imagery is easiest to obtain and manage, such that

527 organisms with relatively flattened body forms will be most tractable (e.g., ticks),

528 although wings of other groups (e.g., mosquitoes and sandflies) may also offer

529 opportunities (Zhou et al. 1985, Godoy et al. 2014, Lorenz et al. 2015). Small size may

530 present some level of challenge, as we will have to manage the complexities of

531 magnification and associated distortion, but our photo apparatus has been designed to

532 allow addition of accessories to facilitate such challenges in imaging. Ticks are probably

533 the most logical next priority, in light of their size and relatively low species diversity (on

534 the order of 700 species; Guglielmone et al. 2010), and considering the growing 
535 appreciation of ticks as significant vectors of many human and livestock diseases

536 (Burgdorfer 1977, Friedhoff 1997, Parola et al. 2005). Agricultural pests will likely offer

537 additional fruitful, tractable, and interesting sets of challenges.

538 In the future, we anticipate deployment of such systems more broadly-e.g., to medical

539 personnel —as well as the broader public, to provide identification services for medically

540 or economically important arthropod groups. The suite of methods described here

541 provides a low-cost avenue to capture of high-quality images of triatomines and is

542 promising to permit specific identification of triatomines. Quite simply, the broader

543 initiative has the potential to open the knowledge of a handful of experts to a much-

544 broader public that may benefit from access to entomological identification services.

\section{Acknowledgments}

546 We thank Lileia Diotaiuti (Fiocruz MG, Brazil), Mitermayer Reis (Fiocruz BA, Brazil),

547 João Aristeu da Rosa (UNESP SP, Brazil), Renata Timbó (UnB, Brazil), Cleber Galvão

548 and Jane Costa (Fiocruz RJ, Brazil), Eduardo Rebollar-Tellez (UANL, Mexico), Luis

549 Alberto Hernandez-Osorio (UABJ, Mexico), and Jorge Lopez-Cardenas (LaESaP,

550 Guanajuato, Mexico) for permitting photography of triatomine collections. We thank

551 Renata Timbó (UnB, Brazil) for helping photograph triatomines at UnB. We thank Perry

552 Alexander, Leonard Krishtalka, and Saralyn Reece-Hardy, for their leadership and

553 guidance in assembling such an interdisciplinary team.

\section{Literature Cited}


555 Acevedo, M. A., C. J. Corrada-Bravo, H. Corrada-Bravo, L. J. Villanueva-Rivera, and T.

556 M. Aide. 2009. Automated classification of bird and amphibian calls using

557 machine learning: A comparison of methods. Ecological Informatics 4:206-214.

558 Allen, W. and E. Rajotte. 1990. The changing role of extension entomology in the IPM

$559 \quad$ era. Annual Review of Entomology 35:379-397.

560 Almeida, C. E., E. Folly-Ramos, A. T. Peterson, V. Lima-Neiva, M. Gumiel, R. Duarte, M.

561 Locks, M. Beltrão, and J. Costa. 2009. Could the bug Triatoma sherlocki be

562 vectoring Chagas disease in small mining communities in Bahia, Brazil? Medical

563 and Veterinary Entomology 23:410-417.

564 Arbuckle, T., S. Schröder, V. Steinhage, and D. Wittmann. 2001. Biodiversity informatics

565 in action: Identification and monitoring of bee species using ABIS. Pages $425-430$

566 Proceedings of the 15th International Symposium on Informatics for

$567 \quad$ Environmental Protection. Metropolis Verlag, Marburg.

568 Arita, H. T., J. A. Christen, P. Rodríguez, and J. Soberón. 2008. Species diversity and

569 distribution in presence-absence matrices: Mathematical relationships and

$570 \quad$ biological implications. American Naturalist 172:519-532.

571 Ballard, D. H. 1981. Generalizing the Hough transform to detect arbitrary shapes.

$572 \quad$ Pattern Recognition 13:111-122.

573 Beard, C. B., G. Pye, F. J. Steurer, Y. Salinas, R. Campman, A. T. Peterson, J. M.

574 Ramsey, R. A. Wirtz, and L. E. Robinson. 2002. Chagas disease in a domestic

575 transmission cycle in southern Texas, USA. Emerging Infectious Diseases 9:103-

576105.

577 Burgdorfer, W. 1977. Tick-borne diseases in the United States: Rocky Mountain spotted

578 fever and Colorado tick fever; a review. Acta Tropica 34:103-126. 
579 Chesmore, D. 2004. Automated bioacoustic identification of species. Anais Da Academia $580 \quad$ Brasileira De Ciencias 76:436-440.

581 Costa, J., L. L. Dornak, C. E. Almeida, and A. T. Peterson. 2014. Distributional potential 582 of the Triatoma brasiliensis species complex at present and under scenarios of $583 \quad$ future climate conditions. Parasites \& Vectors 7:238.

584 Costa, J. and A. T. Peterson. 2012. Ecological niche modeling as a tool for 585 understanding distributions and interactions of vectors, hosts, and etiologic 586 agents of Chagas disease. Advances in Experimental Medicine and Biology $587 \quad 710: 59-70$.

588 Costa, J., A. T. Peterson, and C. B. Beard. 2002. Ecological niche modeling and 589 differentiation of populations of Triatoma brasiliensis Neiva, 1911, the most 590 important Chagas disease vector in northeastern Brazil (Hemiptera, Reduviidae, 591 Triatominae). American Journal of Tropical Medicine \& Hygiene 67:516-520.

592 Cywinska, A., F. F. Hunter, and P. D. N. Hebert. 2006. Identifying Canadian mosquito 593 species through DNA barcodes. Medical and Veterinary Entomology 20:413-424.

594 Dias, J. C. P., A. C. Silveira, and C. J. Schofield. 2002. The impact of Chagas disease 595 control in Latin America: A review. Memórias do Instituto Oswaldo Cruz 97:603596 612.

597 Drew, L. W. 2011. Are we losing the science of taxonomy? As need grows, numbers and $598 \quad$ training are failing to keep up. BioScience 61:942-946.

599 Dujardin, J.-P. and D. E. Slice. 2007. Contributions of morphometrics to medical 600 entomology. Pages 433-445 in M.Tibayrenc, editor. Encyclopedia of infectious 601 diseases: modern methodologies. John Wlley and Sons.

602 Friedhoff, K. T. 1997. Tick-borne diseases of sheep and goats caused by Babesia, 603 Theileria or Anaplasma spp. Parassitologia 39:99-109. 
604 Gaston, K. J. 1992. Taxonomy of taxonomists. Nature 356:281-282.

605 Gaston, K. J. and M. A. O'Neill. 2004. Automated species identification: Why not?

606 Philosophical Transactions of the Royal Society of London B 359:655-667.

607 Godoy, R. E., E. A. B. Galati, P. Cordeiro-Estrela, N. A. De Souza, T. V. Dos Santos, L.

608 C. De Sousa, and E. F. Rangel. 2014. Comparative study of the phlebotomine

609 sand fly species (Diptera: Psychodidae: Phlebotominae) of the genera

610 Nyssomyia Barretto, 1962, Bichromomyia Artemiev, 1991, and Migonemyia

611 Galati, 1995, vectors of American cutaneous leishmaniasis in Brazil. Zootaxa

$612 \quad 3838: 501-517$.

613 Guglielmone, A. A., R. G. Robbins, D. A. Apanaskevich, T. N. Petney, A. Estrada-Peña, I.

614 G. Horak, R. Shao, and S. C. Barker. 2010. The Argasidae, Ixodidae and

615 Nuttalliellidae (Acari: Ixodida) of the world: A list of valid species names. Zootaxa

$616 \quad 2528: 1-28$

617 Gurgel-Gonçalves, R., J. B. Ferreira, A. F. Rosa, M. E. Bar, and C. Galvão. 2011.

618 Geometric morphometrics and ecological niche modelling for delimitation of near-

619 sibling triatomine species. Medical and Veterinary Entomology 25:84-93.

620 Gurgel-Gonçalves, R., C. Galvão, J. Costa, and A. T. Peterson. 2012. Geographic

621 distribution of Chagas disease vectors in Brazil based on ecological niche

622 modeling. Journal of Tropical Medicine 2012:705326.

623 Hebert, P. D. and T. R. Gregory. 2005. The promise of DNA barcoding for taxonomy.

$624 \quad$ Systematic Biology 54:852-859.

625 Hijmans, R. J. 2015. raster: Geographic Data Analysis and Modeling, R package version

626 2.5-2; https://CRAN.R-project.org/package=raster.

627 Ibarra-Cerdeña, C. N., V. Sánchez-Cordero, A. Townsend Peterson, and J. M. Ramsey.

628 2009. Ecology of North American Triatominae. Acta Tropica 110:178-186. 
629 Ibarra-Cerdeña, C. N., A. Zaldivar-Riverón, A. T. Peterson, V. Sánchez-Cordero, and J.

630 M. Ramsey. 2014. Phylogeny and niche conservatism in North and Central

631 American triatomine bugs (Hemiptera: Reduviidae: Triatominae), vectors of

632 Chagas' disease. PLoS Neglected Tropical Diseases 8:e3266.

633 Kang, S. H., W. Jeon, and S. H. Lee. 2012a. Butterfly species identification by branch

634 length similarity entropy. Journal of Asia-Pacific Entomology 15:437-441.

635 Kang, S. H., S. H. Song, and S. H. Lee. 2012b. Identification of butterfly species with a

636 single neural network system. Journal of Asia-Pacific Entomology 15:431-435.

637 Lent, H. and P. Wygodzinsky. 1979. Revision of the Triatominae (Hemiptera,

638 Reduviidae), and their significance as vectors of Chagas' disease. Bulletin of the

639 American Museum of Natural History 163:123-520.

640 López-Cárdenas, J., F. E. González-Bravo, P. M. Salazar-Schettino, J. C. Gallaga-

641 Solórzano, E. Ramírez-Barba, J. Martínez-Méndez, V. Sánchez-Cordero, A. T.

642 Peterson, and J. M. Ramsey. 2005. Fine-scale predictions of distributions of

643 Chagas disease vectors in the state of Guanajuato, Mexico. Journal of Medical

$644 \quad$ Entomology 42:1068-1081.

645 Lorenz, C., A. S. Ferraudo, and L. Suesdek. 2015. Artificial neural network applied as a 646 methodology of mosquito species identification. Acta Tropica 152:165-169.

647 MacLeod, N., editor. 2007. Automated Taxon Identification in Systematics: Theory, 648 Approaches and Applications. CRC Press, Boca Raton.

649 MacLeod, N., M. Benfield, and P. Culverhouse. 2010. Time to automate identification.

$650 \quad$ Nature 467:154-155.

651 Mathis, A., J. Depaquit, V. Dvořák, H. Tuten, A.-L. Bañuls, P. Halada, S. Zapata, V.

652 Lehrter, K. Hlavačková, J. Prudhomme, P. Volf, D. Sereno, C. Kaufmann, V.

653 Pflüger, and F. Schaffner. 2015. Identification of phlebotomine sand flies using 
one MALDI-TOF MS reference database and two mass spectrometer systems. Parasites \& Vectors 8:1-9.

Mayo, M. and A. T. Watson. 2007. Automatic species identification of live moths. Knowledge-Based Systems 20:195-202.

MBI. 2009. Mosquito Barcoding Initiative; http://connect.barcodeoflife.net/group/mosquitobarcoding.

660 Meier, R., K. Shiyang, G. Vaidya, and P. K. Ng. 2006. DNA barcoding and taxonomy in Diptera: A tale of high intraspecific variability and low identification success. Systematic Biology 55:715-728.

Moore, A. and R. H. Miller. 2002. Automated identification of optically sensed aphid (Homoptera: Aphidae) wingbeat waveforms. Annals of the Entomological Society of America 95:1-8.

666 Parola, P., C. D. Paddock, and D. Raoult. 2005. Tick-borne rickettsioses around the 667 world: Emerging diseases challenging old concepts. Clinical Microbiology Reviews 18:719-756.

669 Peterson, A. T., M. Papeş, and J. Soberón. 2008. Rethinking receiver operating 670 characteristic analysis applications in ecological niche modeling. Ecological $671 \quad$ Modelling 213:63-72.

672 Peterson, A. T., V. Sánchez-Cordero, C. B. Beard, and J. M. Ramsey. 2002. Ecologic 673 niche modeling and potential reservoirs for Chagas disease, Mexico. Emerging $674 \quad$ Infectious Diseases 8:662-667.

675 Pinto, I. d. S., B. D. das Chagas, A. A. F. Rodrigues, A. L. Ferreira, H. R. Rezende, R. V. 676 Bruno, A. Falqueto, J. D. Andrade-Filho, E. A. B. Galati, and P. H. F. 677 Shimabukuro. 2015. DNA Barcoding of Neotropical sand flies (Diptera, 
Psychodidae, Phlebotominae): Species identification and discovery within Brazil. PLoS ONE 10:e0140636.

680 Ramsey, J. M., R. Ordoñez, A. Cruz-Celis, A. Alvear, V. Chávez, R. López, J. Pintor, F. stratification of Chagas disease transmission in Oaxaca, Mexico. Medical and Veterinary Entomology 14:30-41.

Ramsey, J. M., A. T. Peterson, O. Carmona-Castro, D. A. Moo-Llanes, Y. Nakazawa, M. Butrick, E. Tun-Ku, K. d. I. Cruz-Félix, and C. N. Ibarra-Cerdeña. 2015. Atlas of Mexican Triatominae (Reduviidae: Hemiptera) and vector transmission of Chagas disease. Memorias del Instituto Oswaldo Cruz 110:339-352.

Ripley, B. D. 1996. Pattern Recognition and Neural Networks. Cambridge University Press, Cambridge.

Schofield, C. J. and C. Galvão. 2009. Classification, evolution, and species groups within the Triatominae. Acta Tropica 110:88-100.

Schroder, S., W. Drescher, V. Steinhage, and B. Kastenholz. 1995. An automated method for the identification of bee species (Hymenoptera: Apoidea). Pages 6-7 Proceedings of the International Symposium on Conserving Europe's Bees. International Bee Research Association.

Soberón, J. and G. Ceballos. 2011. Species richness and range size of the terrestrial mammals of the world: Biological signal within mathematical constraints. PLoS ONE 6:e19359.

Stevenson, R., W. A. Haber, and R. A. Morris. 2003. Electronic field guides and user communities in the eco-informatics revolution. Conservation Ecology 7:3. 
701 Valdez-Tah, A., L. Huicochea-Gómez, J. Ortega-Canto, A. Nazar-Beutelspacher, and J.

702 M. Ramsey. 2015. Social representations and practices towards triatomines and 703 Chagas disease in Calakmul, Mexico. PLoS ONE 10:e0132830.

704 Voltsit, O. V. and I. Pavlinov. 1995. [The use of the methods of geometrical morphometry $705 \quad$ in the systematics of ixodid ticks (Ixodidae)]. Parazitologiia 30:292-301.

706 Wang, J., L. Ji, A. Liang, and D. Yuan. 2012a. The identification of butterfly families 707 using content-based image retrieval. Biosystems engineering 111:24-32.

708 Wang, J., C. Lin, L. Ji, and A. Liang. 2012b. A new automatic identification system of 709 insect images at the order level. Knowledge-Based Systems 33:102-110.

710 Watson, A. T., M. A. O'Neill, and I. J. Kitching. 2003. Automated identification of live 711 moths (Macrolepidoptera) using Digital Automated Identification SYstem (DAISY).

$712 \quad$ Systematics and Biodiversity 1:287-300.

713 Weeks, P. J. D., I. D. Gauld, K. J. Gaston, and M. A. O'Neill. 1997. Automating the 714 identification of insects: A new solution to an old problem. Bulletin of $715 \quad$ Entomological Research 87:203-211.

716 Weeks, P. J. D., M. A. O’Neill, K. J. Gaston, and I. D. Gauld. 1999. Automating insect 717 identification: Exploring the limitations of a prototype system. Journal of Applied $718 \quad$ Entomology 123:1-8.

719 Wu, T.-F., C.-J. Lin, and R. C. Weng. 2004. Probability estimates for multi-class 720 classification by pairwise coupling. Journal of Machine Learning Research 5:975$721 \quad 1005$.

722 Yang, H.-P., C.-S. Ma, H. Wen, Q.-B. Zhan, and X.-L. Wang. 2015. A tool for developing 723 an automatic insect identification system based on wing outlines. Scientific $724 \quad$ reports 5:12786. 
725 Zhou, Y.-H., L.-B. Ling, and F. J. Rohlf. 1985. Automatic description of the venation of 726 mosquito wings from digitized images. Systematic Biology 34:346-358. 
727 Figure 1. Photographs of the apparatus designed for capture of high-quality images of 728 triatomine bugs for this project. The top image shows a view from above, while the 729 bottom image shows a lateral view, showing the lighting ring and an insect (not a 730 triatomine) impaled on the pin (note that the pin does not protrude through the dorsum 731 and thus is not visible in the image).

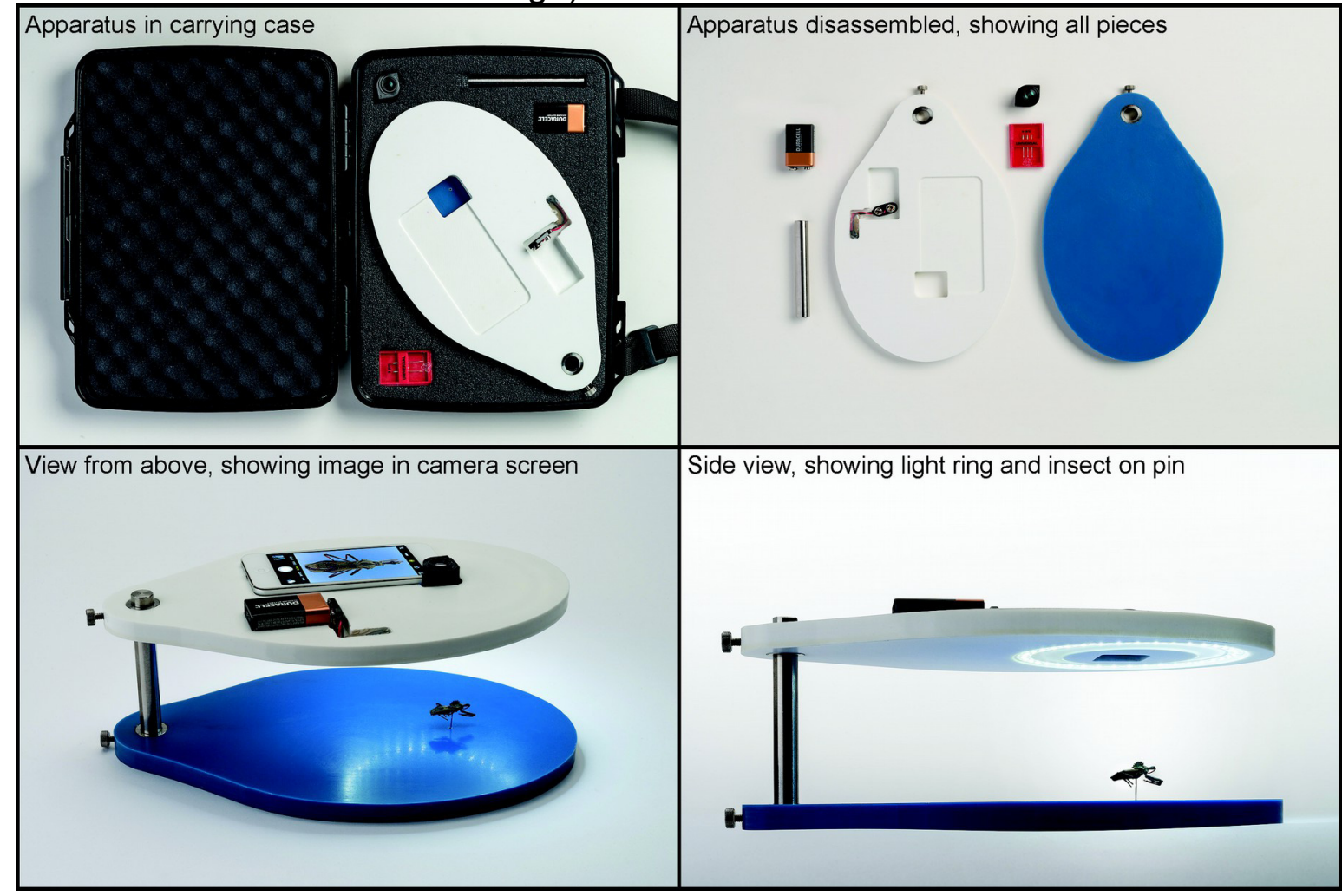


732 Figure 2. Summary of major steps in the processing of an example image of an

733 individual of Triatoma brasiliensis. Panels from left to right: (1) raw image, (2)

734 background removed to create a binary image, (3) legs and antennae removed and

735 edge identified, (4) insect body filled and landmarks added, and (5) final image with

736 landmarks overlaid.

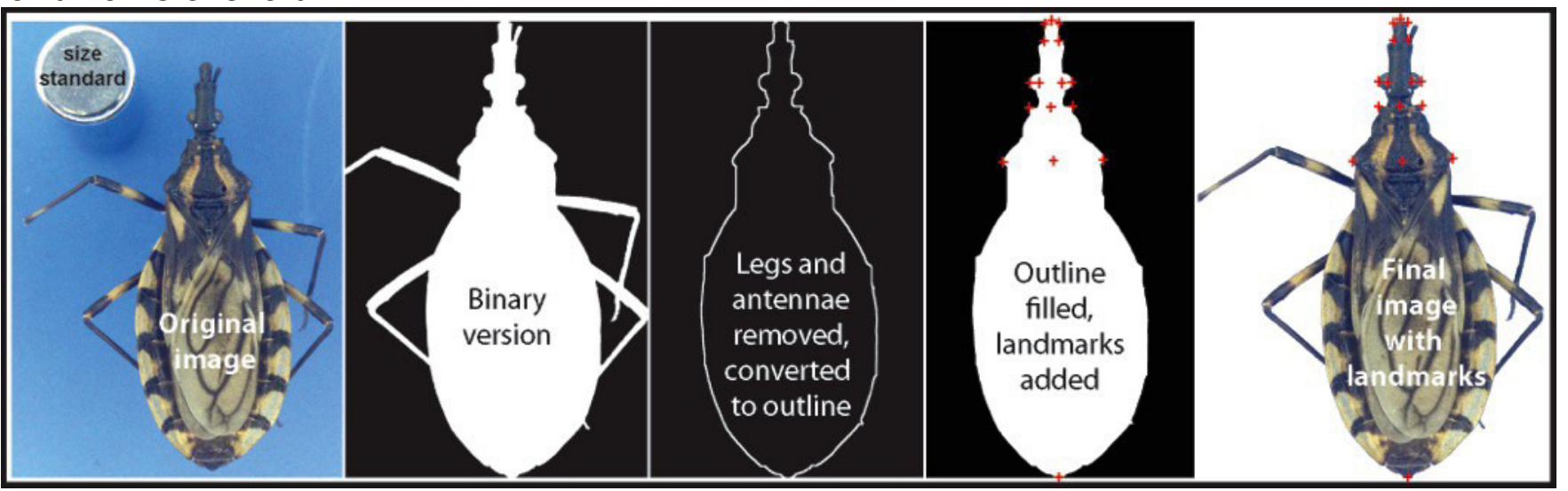


737 Figure 3. Map of species richness among the 39 triatomine species that are the focus of 738 this analysis across Brazil, and 12 species across Mexico, each with three example sites 739 and their corresponding triatomine faunas.
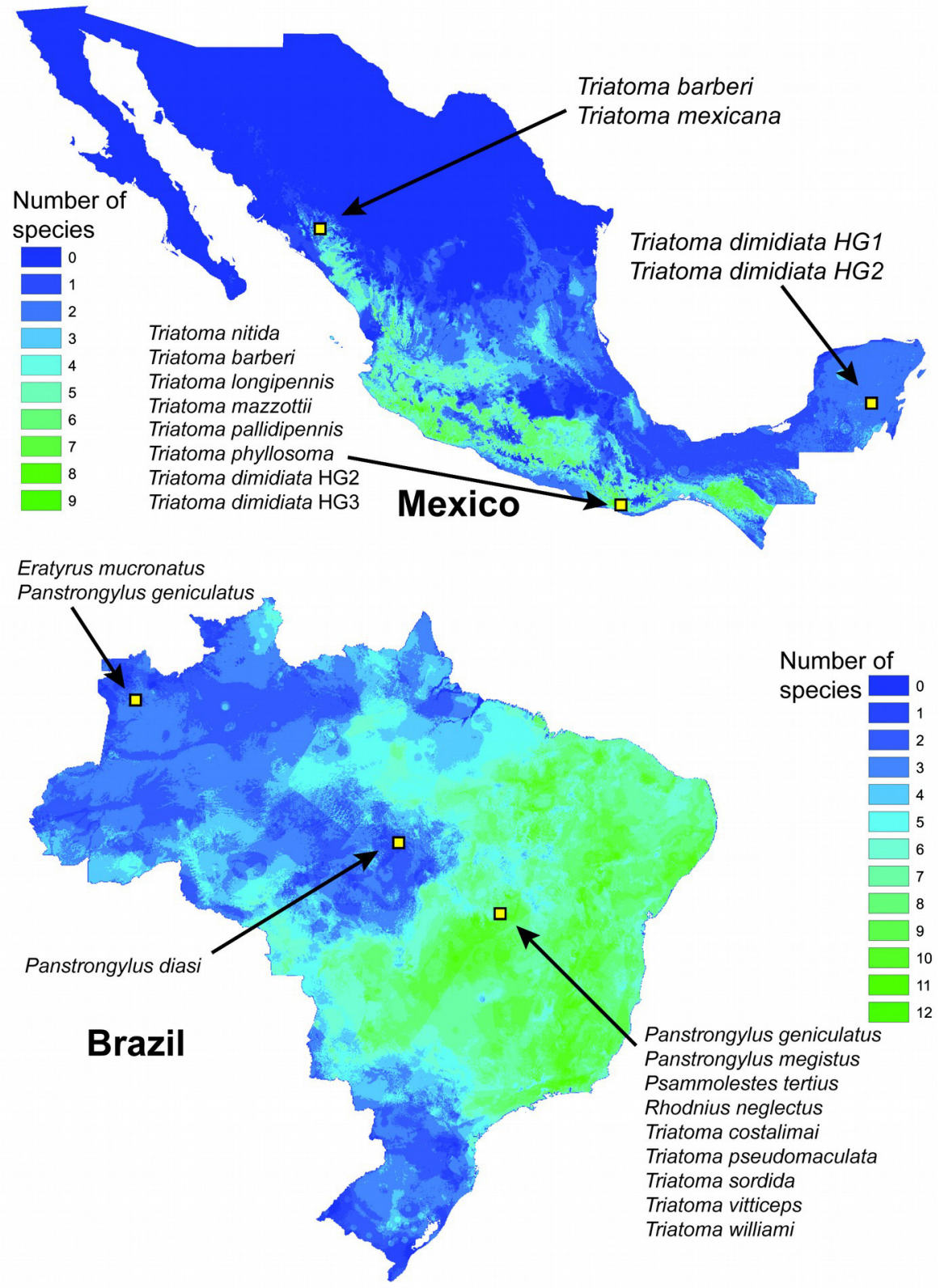
740 Figure 4. Illustration of the spread of different species of Brazilian and Mexican

741 triatomine bugs in a morphological space defined by the first two principal components

742 (Brazil: component $1=44.0 \%$ of overall variation, component $2=21.8 \%$ of overall

743 variation; Mexico: component $2=18.7 \%$ of overall variation, component $3=12.5 \%$ of

744 overall variation - note that species separated best in this space of component 3 vs

745 component 2), summarizing all of the measurements used in this study. The top panel

746 shows the distribution of all of the genera except for Triatoma, and the bottom panel

747 shows the distribution of Triatoma species only, as a zoom of the central part of the top

748 panel.

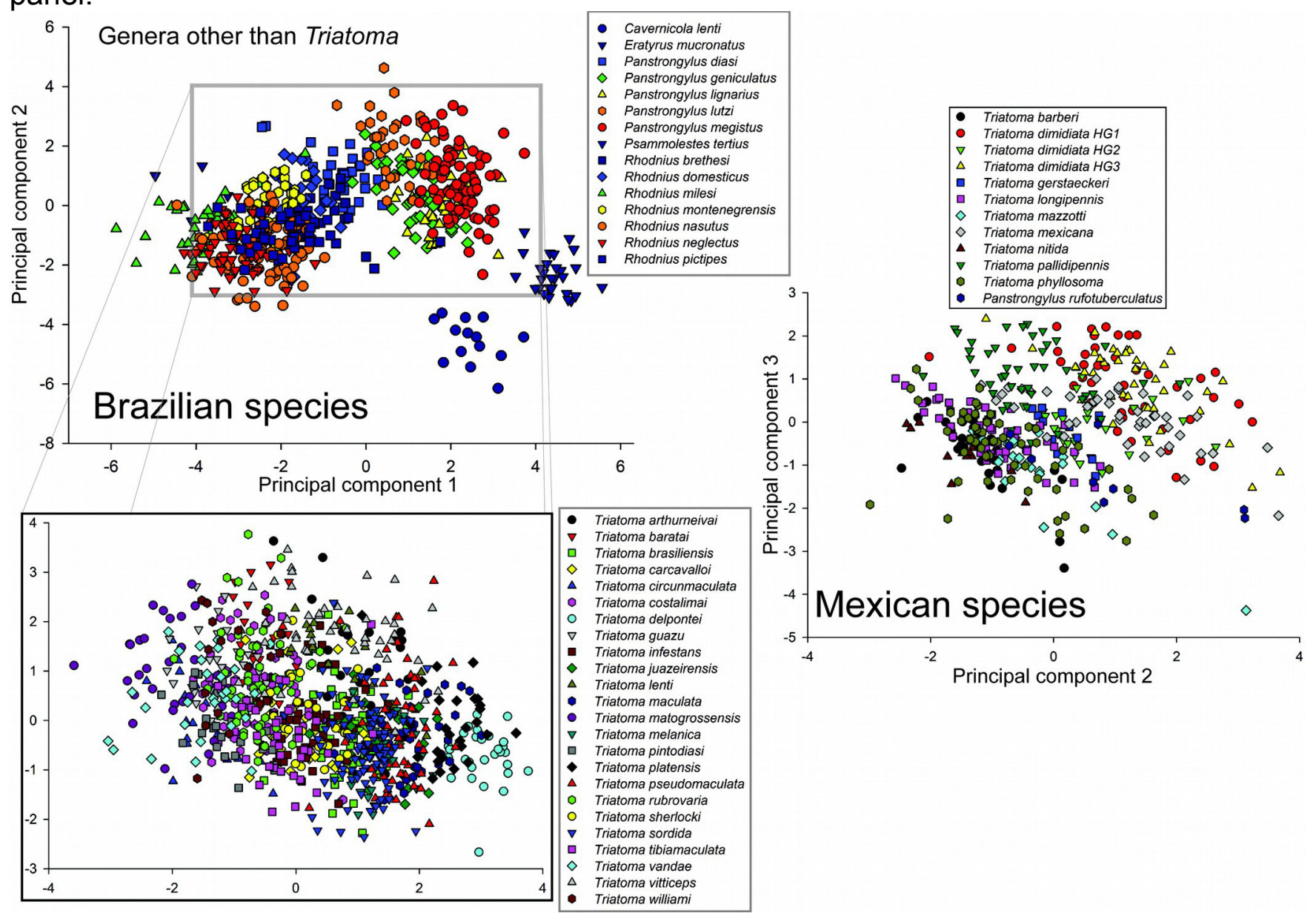


749 Figure 5. Summary of classification success rates for 200 random combinations of 750 numbers of species of 2-37 species for Brazilian triatomine faunas and 2-11 species for 751 Mexican triatomine faunas (open symbols). Also shown are success rates based on 752 real-world species combinations at the testing sites (gray-filled symbols). Error bars are 753 shown to indicate standard deviations for each fauna size.

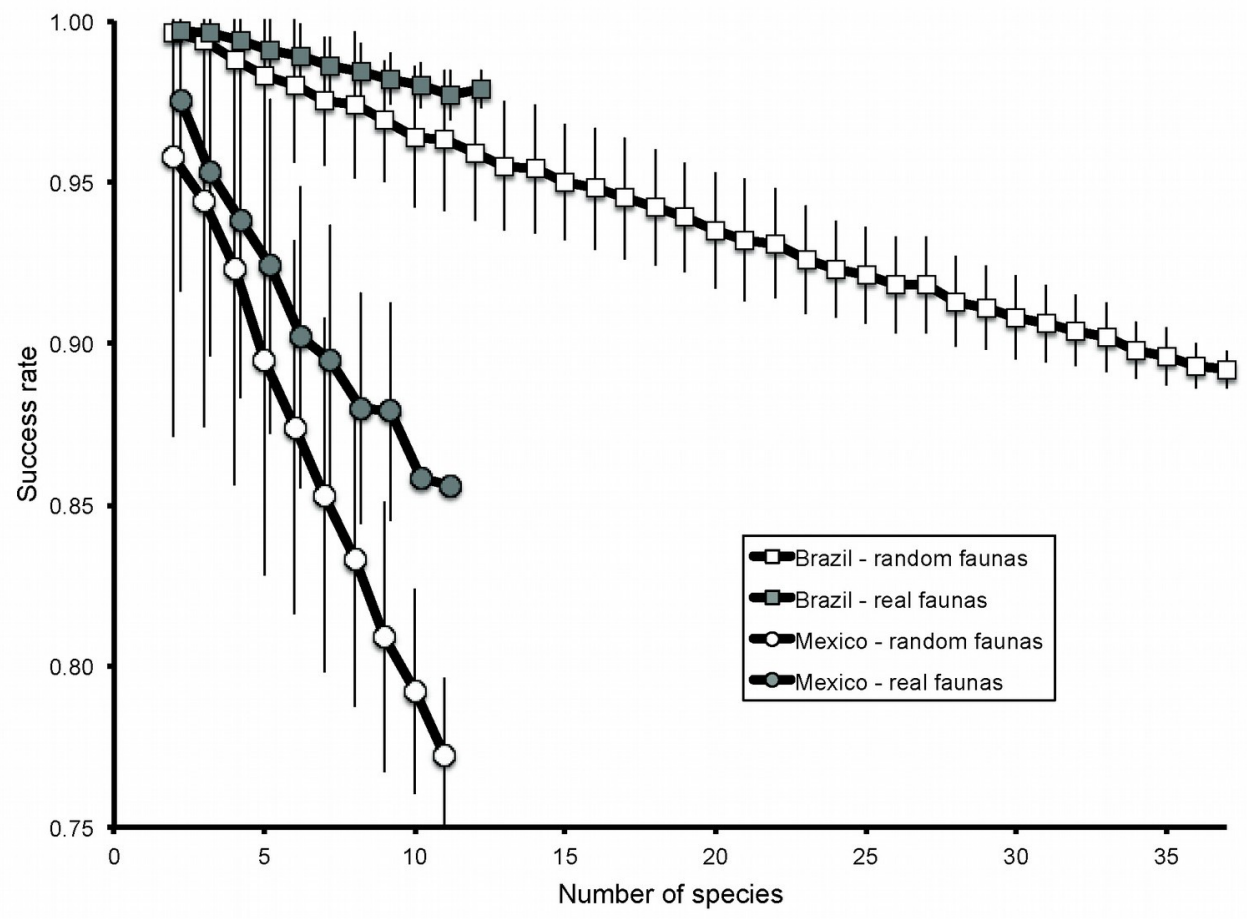


754 Table 1. Summary of species analyzed, sample sizes of photographs, and identification

755 success rates, for the 39 species of Brazilian triatomine bugs analyzed in this study.

\begin{tabular}{lcc} 
Species & $\begin{array}{c}\text { Sample } \\
\text { size }\end{array}$ & \begin{tabular}{c} 
Success rate \\
\hline Cavernicola lenti Barrett \& Arias, 1985
\end{tabular} \\
\hline Eratyrus mucronatus Stål, 1859 & 15 & 93.3 \\
\hline Panstrongylus diasi Pinto \& Lent, 1946 & 10 & 80.0 \\
\hline Panstrongylus geniculatus (Latreille, 1811) & 30 & 96.7 \\
\hline Panstrongylus lignarius (Walker, 1873) & 45 & 93.3 \\
\hline Panstrongylus lutzi Neiva \& Pinto, 1923 & 28 & 85.7 \\
\hline Panstrongylus megistus Burmeister, 1835 & 34 & 88.2 \\
\hline Psammolestes tertius Lent \& Jurberg, 1965 & 84 & 91.7 \\
\hline
\end{tabular}

Rhodnius brethesi Matta, 1919

28

Rhodnius domesticus Neiva \& Pinto, 1923

28

Rhodnius milesi Carcavallo, Rocha, Galvão \& Jurberg, 2001

$27 \quad 96.3$

Rhodnius montenegrensis Rosa et al. 2012

37

89.2

Rhodnius nasutus Stål, 1859

39

84.6

Rhodnius neglectus Lent, 1954

73

Rhodnius pictipes Stål, 1872

60

82.2

43

Triatoma arthurneivai Lent \& Martins, 1940

32

83.3

Triatoma baratai Carcavallo \& Jurberg, 2000

Triatoma brasiliensis Neiva, 1911

Triatoma carcavalloi Jurberg, Rocha \& Lent, 1998

Triatoma circummaculata (Stål, 1859)

73

43

95.3

Triatoma costalimai Verano \& Galvão, 1958

Triatoma delpontei Romana \& Abalos, 1947

Triatoma guazu Lent \& Wygodzinsky, 1979

Triatoma infestans (Klug, 1834)

29

64

38

21

78.1

Triatoma juazeirensis Costa \& Felix, 2007

Triatoma lenti Sherlock \& Serafim, 1967

Triatoma maculata (Erichson, 1848)

Triatoma matogrossensis Leite \& Barbosa, 1953

Triatoma melanica Neiva \& Lent, 1941

Triatoma pintodiasi Jurberg, Cunha \& Rocha, 2013

Triatoma platensis Neiva, 1913

Triatoma pseudomaculata Correa \& Espínola, 1964

Triatoma rubrovaria (Blanchard, 1843)

Triatoma sherlocki Papa, Jurberg, Carcavallo, Cerqueira \& Barata, 2002

Triatoma sordida (Stål, 1859)

Triatoma tibiamaculata (Pinto, 1926)

63

29

29

82.8

76.6

86.8

Triatoma vandae Carcavallo, Jurberg, Rocha, Galvão, Noireau \& Lent, 2002

Triatoma vitticeps (Stål, 1859)

Triatoma williami Galvão, Souza \& Lima, 1965

28

54

21

19

39

32

29

25

27

55

54

31

96

$41 \quad 92.7$

86.8

85.7

85.7

86.7

64.3

83.3

81.0

78.9

89.7

75.0

79.3

88.0

74.1

70.9

59.3

93.5

81.2

29

$47 \quad 85.1$

69.0

17

70.6 
756 Table 2. Summary of species analyzed, sample sizes of photographs, and identification 757 success rates, for the 12 species of Mexican triatomine bugs analyzed in this study.

\begin{tabular}{lcc}
\multicolumn{1}{c}{ Species } & Sample size & Success rate \\
\hline Panstrongylus rufotuberculatus (Champion, 1899) & 7 & 100.0 \\
\hline Triatoma barberi Usinger, 1939 & 29 & 72.4 \\
\hline Triatoma dimidiata (Latreille, 1811) HG1 & 44 & 70.5 \\
\hline Triatoma dimidiata (Latreille, 1811) HG2 & 30 & 76.7 \\
\hline Triatoma dimidiata (Latreille, 1811) HG3 & 40 & 82.5 \\
\hline Triatoma gerstaeckeri (Stål, 1859) & 12 & 83.3 \\
\hline Triatoma longipennis Usinger, 1939 & 51 & 72.5 \\
\hline Triatoma mazzottii Usinger 1941 & 22 & 77.3 \\
\hline Triatoma mexicana (Herrich-Schaeffer, 1848) & 45 & 80.0 \\
\hline Triatoma nitida Usinger, 1939 & 15 & 46.7 \\
\hline Triatoma pallidipennis Stål, 1872 & 43 & 90.7 \\
\hline Triatoma phyllosoma (Burmeister, 1835) & 58 & 46.6 \\
\hline
\end{tabular}

